\title{
Discussion on Different Types of Game User Interface
}

\author{
Yunsong $\mathrm{Hu}^{1, \text { a }}$ \\ ${ }^{1}$ college of Electronical and Information Engineering, Chengdu University of Information Technology, \\ Chengdu 610225, China.
}

a532820458@qq.com

Keywords: game user interface, WIMP, comparisons.

\begin{abstract}
With the development of computer industry, the user interface has become the international research direction. It is believed that there are some important factors in the user interface design, such as window, icon, pointing device, and menu, will influence the games. The sum of these factors is called WIMP. At the same time, some other game elements will also make impacts to the gaming experience, including player situation, navigation system, item bar, etc. This paper will introduce some different types of games, including First-person shooting game, Role-playing game, and Real-time strategy game. The following parts will analyze the reasons why there are some differences in user interface among these types of games.
\end{abstract}

\section{Introduction}

In recent years, with the rapid development of software engineering and information engineering, the promotion of new generation of computer technology research and the rapid development of network technology, the user interface design and development has become the most active research direction in the international computer industry. More and more mechanical engineers, software designers, electronic engineers and industrial designers who work for the man-machine interface has made a significant contribution.

Man-machine interface is the user and the computer interface, which contact users with the computer hardware, software, a comprehensive environment. If designers need to design a friendly man-machine interface, they should understand the characteristics of the man-machine interface. A perfect interface design should be aware of its ease of usability, flexibility, complexity and reliability [1]. Considering the user's characteristics, ability, knowledge level is different. So, there should be different interface forms for different users. Users can customize and modify the interface as needed. The system should provide different levels of system response information according to the user's wishes and needs, including feedback information, promotion information, helping information, error information, etc.

As important as the game user interface design. Game players also need a layer to contact with the whole game playing. With the development of game user interface design industry, there are many different types of user interface.

Different types of games have different user interface which can affect players' experience when they are playing games. This paper will introduce the UI design of different game types and analyze and compare the differences between early and modern Games.

\section{Background}

Video game machine was produced in early 70s.NolanBushnell who was still studying in MIT designed the first business game machine in the world, the arcade game called COMPUTER SPACE. It means the beginning of the game industry, because it is the first specialized game machine. It is the first tool to allow the public to contact games.

In the game industry, as Ernest Adams said that user interface is the core mechanics which mediates the game and the player. It turns information as graphics on the screen and sound from the 
speakers to the player in the game. It also turns the player's button presses and movement on the keyboard or controller into actions within the context of the game. It is one of the most important factors in the games [2]. The user interface which provides visual feedback is often referred to as the virtual interface. The virtual interface is a layer between the game and the physical input and output from and to the players. It transforms the core mechanism into visual, audio, or physical cues that players can understand. This is also known as a "HUD” (Heads-Up Display). Because it shows the players the information which need to play in the game, like the aircraft on the HUD display important information in the flight [2].

The game user interface has made a great progress in the past 50 years. In the early HUD, the number was represented as score, time, or number as lives. Based on Csikszentmihalyi's (1992) theory, Jones (1998) considered a list how they are manifested in games [3]. With the modern information technology improving, Game designers has begun to focus on cinematic experiences, as players will have a strong sense of participation and enjoyment. Games have let more factors of game user interface transmit information to players, like sound, vibration, and the character models.

On the other hand, cognitive psychology, in a broad sense, is about the psychology of cognition. Human mainly understand the objective things through the senses, perception, attention, memory, thinking imagination[4].Understanding how the user awareness affects game interface design is how to eliminate the gap between the game interface and the user model. Try to eliminate the frustration of the user and eliminate the confusion, confusion, or resistance to the game interface [5]. The main goal of the interface design is developing an interactive system which can cause a positive response from the user. Good interface design allows users to enjoy the use of the process. How to design a user-specific emotional response to the interaction system is the designer should be concerned about the problem. This interactive system encourages users to take the initiative to learn, play, interact and can inspire creativity. These factors that can cause a user-specific emotional response are called emotional factors in the design.

The user's affective experience is paramount. The positive effects associated with the game can take many forms including satisfaction, sense of accomplishment, entertainment or excitement [3]. The main motivation of users is to achieve this goal, and to experience the impact of positive desire.

\section{WIMP interface style}

The design of the existing computer game user interface is mostly similar to the WIMP (Window-Icon-Menu-Pointing Device) interface style in the man-machine interface design [6].

Window. The window in the game will not appear on the screen. But through other ways to show up. The general way is to use the button link, shortcut keys calling out, the program which is in a specific situation automatically showing up. The designer should take care the window that display the main screen of the game screen relationship, the size of the window, the position of default showing up and transparency which will affect the game.

Icon. There are many forms of icons, which can be a true representation of the object replaced by the icon, or it can be highly stylized. Icons can also be used to represent other items in the game, such as props in the game, character status, character performance, and so on. Or even any symbol, but in the design of the icon should be considered by the user's own meaning for itself.

Pointing Device. The design of the pointing device in the game interface design is also a very important part. Different shapes of the pointing device is often used to distinguish different patterns. The pointing device can also be used to tell the user that the system is working. For example, in WARCRAFT, when the unit is selected, the pointing device is green and the pointing device is yellow when the friendly unit is selected. When the enemy unit is selected, the pointing device is red. When the player chooses to attack, or follow the command, the pointing device will become a circle.

Menu. A menu is an interactive technique which is common in many non-Windows systems, allowing the user to select an operation or service which is executed by the system at a setting time. The menu is the orderly operation table for the user to browse and providing a message prompt. This 
means that the name of the command in the menu should be meaningful and it can provide information.

\section{Examples}

\section{First-person shooting game (FPS)}

FPS is a shooting game based on the subjective perspective of the players. Players can personally experience the visual impact of the game. This greatly enhances the game's initiative and realism.

Counter-Strike. Counter Strike was originally Valve's game Half-Life which is one of the game module made by Minh Lee and JessCliffe. After that, the module was purchased by Valve and two producers continue to work in Valve.

The health and armor icon is located at the bottom left of the screen. This is represented by a small yellow cross and shield icon and next to a numeric value, which represents your current life and armor remaining number. The ammo and money icon is located at the bottom right. When the game player is attacked by enemy, the cursor will display the red. This suggests that players are being attacked and their health is declining. The red bar shows the direction that game player was attacked.

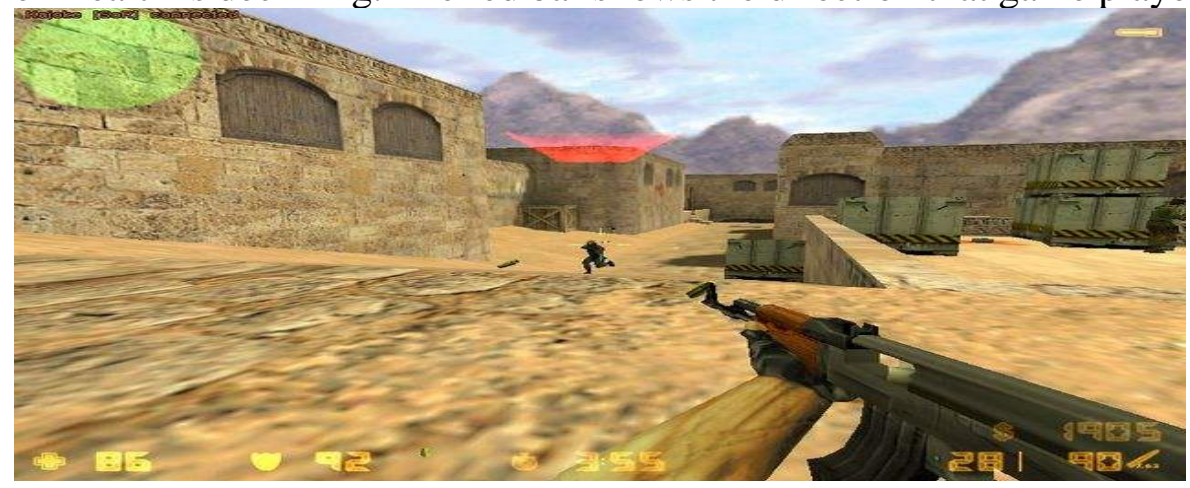

Fig. 1 Counter Strike (http://www.duote.com/soft/3691.html)

Call of Duty: Modern Warfare 3.Call of Duty 3 is a first-person shooter game developed by Treyarch, Activision, which was released in November 2006. Ben as the third series of the call of duty.

This game display important information and missions as text size and font style on the top left of the screen. Many visual text representations promote certain information. This factor enable players more convenience and easier to understand what they should do or what they need. The font size is used to distinguish the more important text information, as the information about the player's target is displayed in the bottom left of the screen. The words of your team allies are located at the bottom of the screen which give players a detailed description of the activities around them, as shown in Figure 2.

The mission objectives are much larger and more explicit. This information is more important than the text (bottom) about the spoken words by the allies.

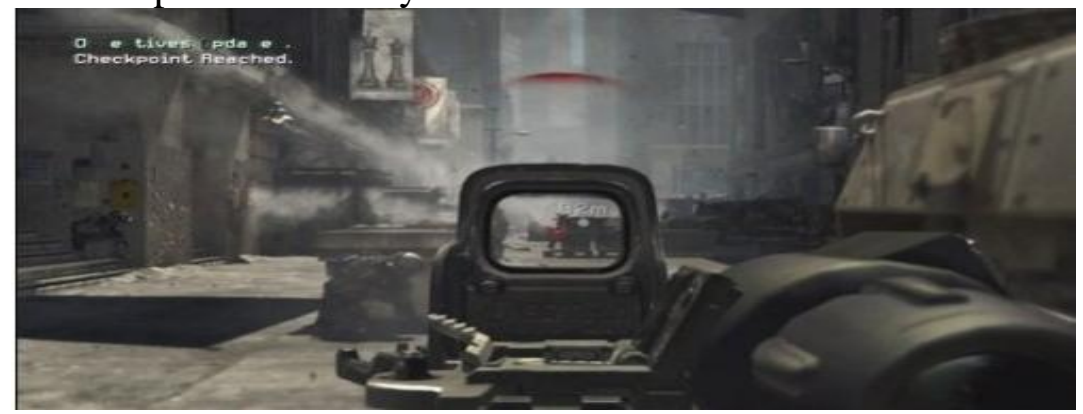

Fig. 2 CallofDuty 3 (http://baike.sogou.com/v319658.htm?ch=ch.bk.innerlink) 


\section{Role-play game (RPG)}

In the game, the player plays the role of the game in a realistic or fictional world. Players play one or more roles and act in a structured way through many actions. The success or failure of a player in the game depends on the formal system of a rule or a course of action.

The Elder Scrolls. Players can interact with the virtual world in The Elder Scrolls V (primarily pc-npc interactions). There are hundreds of places to explore in this vast world, and a large variety of characters and objects to interact with. Therefore, the user interface is one of the most important factors for players to interact with virtual world in the game. As for most RPG game, Health Point display as a red bar, magic point display as a blue bar and physical power display as a green bar. Sometimes the system may hide this information to make players 'visual more clear, if players switch to the attack mode, the information will show up to remind players to notice their situation.

The choice of navigation technology depends on the size of the virtual world and the user's psychology. Users can access and use navigation in the virtual world, from small cities to big powers. Usually, RPG games use a small map in the corner of the screen to guide players. In The Elder Scrolls $\mathrm{V}$, system use a stripe with direction on it to lead users. Designers always use different navigation techniques, to leave the users to choose their favorite style, as shown in Figure 3.

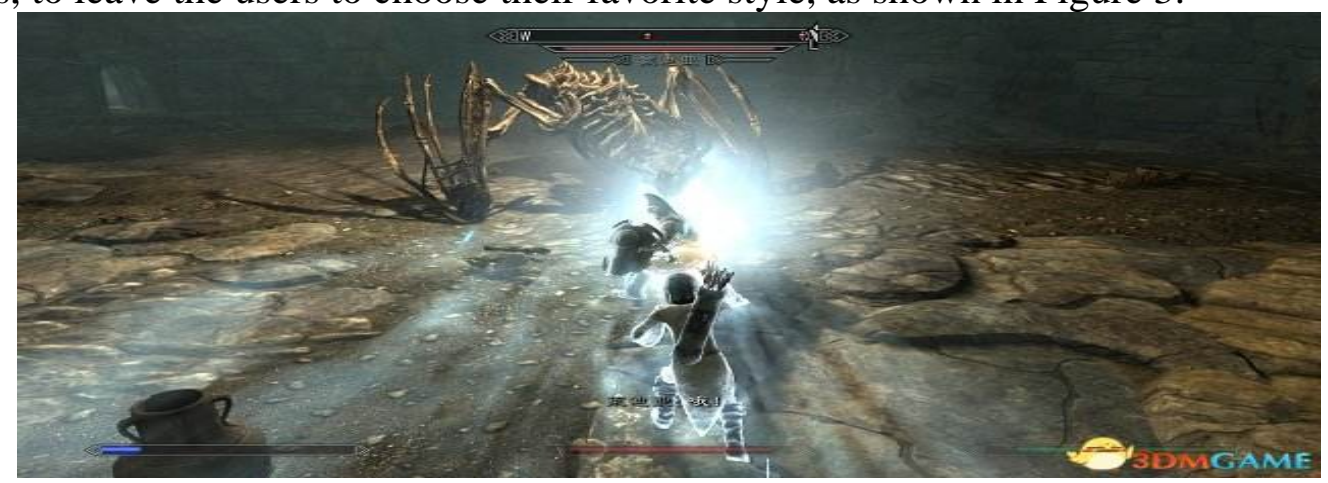

Fig. 3 The Elder Scrolls V (www.3dmgame.com)

\section{Real-time strategy game (RTS)}

The game is instant. Players often play the general in the game. Players usually control the army against AI or other players. There is a big difference between the RTS game and the normal strategy game. It makes people feel pressed for time. The actions of players and opponents are not limited by the turn. Therefore, reaction time is as important as quick action and strategic thinking. The theme of the RTS game in a large extent affect the game interface design.

Warcraft. Window mode is an appropriate choice for complex information in the game. Put the information to be implemented in the window. System menu is on the top of the screen, game display in the middle of the part of the screen, the following part of screen is the operation of the game command button and some information window that players need to pay attention to.

In the game, the player can only see the enemy's life value, and other detailed information, such as the enemy equipped with props and magic values, as well as the character attributes cannot be displayed. At this point, the bottom part of the window screen area will display this information, of course, on the permission is that the mouse should select the unit which user want to know some information, as shown in Figure 4. 


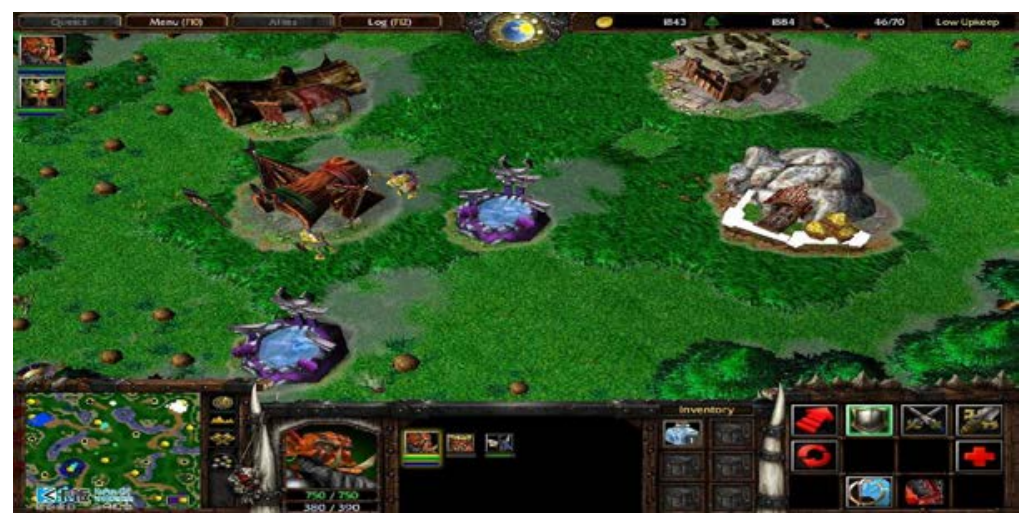

Fig. 4 Warcraft 3 (www.orsoon.com)

\section{Discussion}

Different types of games have different user interfaces. Here are some reasons why their interface has difference.

Players' situation. Most FPS games only display very less information. The interface displays minimal and less information about the current player's health, weapons, and ammo. The game interface does not need to display a lot of information to the player at the moment. Players not only need to play games crazily, but also pay more attention to their situation changes. With the change of color and shape to attract the game player is the most effective method, such as when player is close to death, the color of health bar will turn to red and its size could change as for warning game players.

And in some RPG games, In the game screen, there are important information about their characters in games, such as life, magic, abnormal situation, skills, etc. These factors are more important than FPS games. Because players need to focus on the situation of characters in games at anytime. Usually, the shape of health bar or magic bar is designed as the style accord with this game itself. For example, in the game Castlevania--Lords of Shadow, the health and magic bar was designed as the Gothic Style to attract players easier.

A good player interface of RTS game will make the game easier to organize and simplify. So mostly, the situation bar like health, contribution time and experience are designed very simple but clear. This is because that time is very short for player to think over. So the design of their situation of armies must be simple and clear.

Navigation. In many FPS games, the navigation map is blurred and covered on the main game area, which means that the player must be able to check the map when the player is safe. Or using transparent maps to cover the main game displaying area. Generally, this is an ideal way to allow players to check map information without affecting by emergency around them. But the navigation map still need to hide some information to increase the playability of games. Such as enemies' location.

As for most RPG games, the screen should show all the information the player cares about, such as the life of the characters, the location of the characters, the location of the enemy, the enemy's life, the enemy's attributes, NPC, map information, etc. As mentioned above, so much information need to be displayed on a screen. Linking buttons and narrowing icons are more practical. In addition, designing a shortcut buttons to display the desired information is a good choice. However, in games, players are often confused because they can't find the correct directions. At the time, the game usually shows a simulated compass on the screen to lead the direction.

Navigation map is the most important factor in the RTS game. It is a main guide for player to finish their mission in the game. Mostly, there might be some fog on the map, only display the players' army information and the resources information; hiding enemies' information to balance game playing for all players in the game.

Item bar. The interface design of FPS games is simple, and follow the "fool" principle. The interface should be as simple as possible. So the item bar should be much simpler than any other 
interface factors. Because players want to play smoothly, they need to know their situation quickly and accurately.

It is difficult to design an object bar system for handling complex item in an easy way to manage them. The classical solution of item bar is to divide the player's item bar into a series of squares. The question which need to be considered is the repeated placement of some items and whether the grid of different sizes is identical. Currently, the design of the item bar is all 2D. It seems that all designers forget that the storage box is a 3D space. Maybe it is a good choice to set a 3D item bar in RPG games in the future.

For the same item, most RTS games use pictures instead of item. Using the number showing in the picture to represent the quantity of the item. Objects are measured as little as possible. For example, all objects are represented only by numbers rather than a dedicated metric unit. Players are confused by the presence of many metrics.

\section{Conclusion}

It is important to explore the factors that the game user interface is a tool to contact players and games. The design of game user interface consists of window, icon, pointing device and menu.

This paper analyzes the basic element of user interface in different types of game. Most FPS games use the clear number or words to show players situation and use the red bar to show the critical event. Some RPG games use the long bar to display health, magic or power numerical value. RTS game use changeless interface, mostly putting the important information at the bottom of the screen.

There are some reason why different game using different user interface. It is suggested that players situation is the most significant element in FPS and RPG games. The navigation is the main guide in RTS game for players. It is difficult for a designer to project a perfect item bar, as a classical item bar is not easy for players to manage their items.

In the future research, a thorough understanding of the game will make a major contribution to the research of user interface designs. Designers should pay more attention on physiology and psychology. And then they should analyze what players need in the game playing.

\section{References}

[1] Visual thinking, Aesthetic Intuition, Psychology---Rudolf Arne, Teng ShouYao, Sichuan Renmin Press , 1998.3

[2] Video Game User Interface Design---Jack Campbell《Uwplatt Edu》 .

[3] Effective Affective User Interface Design in Games---Daniel Johnson and Janet Wiles 《Ergonomics》2003.46 (13-14) :1332-1345

[4] Cognitive Psychology---Wang Yan, Wang AnSheng, Peking University Press, 1992.10

[5] Design Psychology ---Donald Norman, Beijing CITIC Publishing House, 2003

[6] Hartson HR.Human-computer interaction: interdisciplinary roots and trends J.The Journal of System and Software. 